\title{
Health monitoring of web plastifying dampers subjected to cyclic loading through vibration tests
}

\author{
Amadeo Benavent-Climent Antolino Gallego \\ Liliana Romo-Melo Leandro Morillas
}

\begin{abstract}
This article investigates experimentally the application of health monitoring techniques to assess the damage on a particular kind of hysteretic (metallic) damper called web plastifying dampers, which are subjected to cyclic loading. In general terms, hysteretic dampers are increasingly used as passive control systems in advanced earthquake-resistant structures. Nonparametric statistical processing of the signals obtained from simple vibration tests of the web plastifying damper is used here to propose an area index damage. This area index damage is compared with an alternative energy-based index of damage proposed in past research that is based on the decomposition of the load-displacement curve experienced by the damper. Index of damage has been proven to accurately predict the level of damage and the proximity to failure of web plastifying damper, but obtaining the load-displacement curve for its direct calculation requires the use of costly instrumentation. For this reason, the aim of this study is to estimate index of damage indirectly from simple vibration tests, calling for much simpler and cheaper instrumentation, through an auxiliary index called area index damage. Web plastifying damper is a particular type of hysteretic damper that uses the out-of-plane plastic deformation of the web of I-section steel segments as a source of energy dissipation. Four l-section steel segments with similar geometry were subjected to the same pattern of cyclic loading, and the damage was evaluated with the index of damage and area index damage indexes at several stages of the loading process. A good correlation was found between area index damage and index of damage. Based on this correlation, simple formulae are proposed to estimate index of damage from the area index damage.
\end{abstract}

\section{Introduction}

Traditional seismic design approach relies on the inelastic deformation of particular zones of the structure to dissipate most of the energy input by the earthquake (commonly, beam-ends and column-ends on momentresisting frames). In contrast, in the passive control systems, this energy is delivered to special devices called seismic dampers.

In the last few decades, passive control has become a popular tool for protecting buildings against earthquakes and preventing or limiting the damage (plastic strains) to the main structure. These systems have many advantages: (1) the inelastic deformations are concentrated in the seismic dampers and the damage in the parent structure can be drastically reduced or eliminated; (2) the addition of damping reduces the lateral displacements of the structure, which also reduces damage to nonstructural elements; and (3) by strategically locating the seismic dampers, their inspection, repair, and/or replacement following an earthquake can be carried out with minimal cost and without interrupting occupancy. Passive damping technologies have been increasingly taken into consideration in the United States after the Northridge earthquake (1994). 
Likewise, since the Kobe (1995) earthquake in Japan, more buildings have been designed to include dampers. ${ }^{1,2}$ Novel research in structural design to control the response to dynamic loads ${ }^{3}$ and new options for passive, semiactive, and active control ${ }^{4}{ }^{6}$ have been proposed in recent years to improve the performance of structures under dynamic loads.

A typical building structure with passive control systems consists of a main frame designed for sustaining mainly the gravity loads and a series of special energy dissipating devices (EDDs), also called dampers, whose main role is to dissipate most of the energy input by the earthquake, as shown in Figure 1. Several mechanisms have been used as EDDs for passive energy dissipation, including metal yielding, phase transformation of metals, friction sliding, fluid orificing, and deformation of viscoelastic solids or liquids. ${ }^{79}$ Among the different EDDs for passive control of structures, the so-called hysteretic damper is one of the most widely used. The source of energy dissipation in hysteretic dampers is the plastic deformation of metals (commonly steel), for which reason they are also known as metallic dampers.

When designing structures with this type of EDD, the connection of the element to the rest of the structure is designed so that it remains elastic (with no damage) for the maximum feasible force that can be sustained by the damper itself; therefore, the damage in the connection is not a concern. However, because inelastic strains in the material involve damage, evaluating the health of the hysteretic damper after a seismic event is a matter of great concern. Furthermore, the same geometry and type of steel is used for all the EDDs (I-section steel segments) installed in the buildings. The lateral strength required in each story is adjusted by varying the

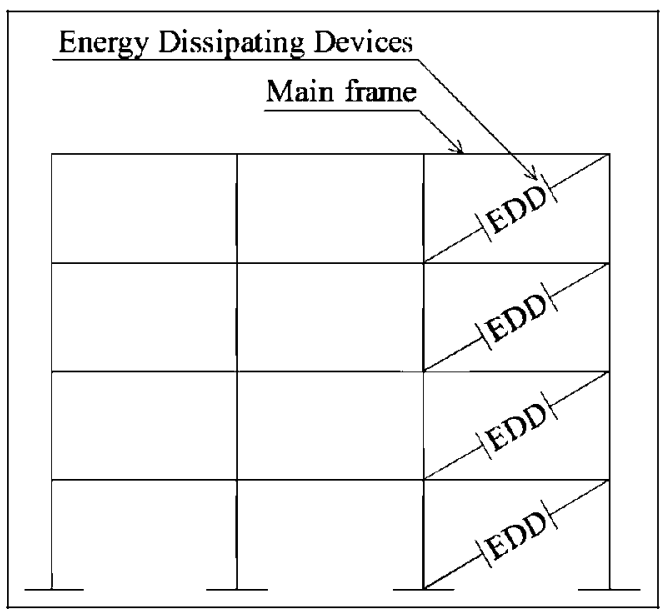

Figure I. Basic configuration of a structure with energy dissipating devices. number of I-section steel segments, but not the characteristics of the I-section steel segment.

For minor or moderate earthquakes (with a probability of exceedance of $50 \%$ and $20 \%$, respectively, in 50 years), the main frame of a structure with hysteretic dampers is commonly designed to remain within the elastic range (i.e. undamaged), and most of the seismic energy input by the earthquake is assumed to be dissipated through plastic deformations on the hysteretic dampers. Minor to moderate earthquakes can occur several times during the lifetime of the structure and usually do not exhaust the energy dissipation capacity of the hysteretic dampers, though they do cause damage. Hysteretic dampers may be effective even after a major earthquake because their ultimate energy dissipation capacity is commonly very large. Hysteretic EDDs need not be replaced after a minor/moderate or even after a large earthquake provided that their health (level of damage) can be reliably evaluated. Such evaluation cannot be based on simple visual inspection because the damage caused by the plastic deformation of the steel is not visible until the element is on the brim of failure.

Past research ${ }^{10}$ has shown that the level of damage and the proximity to failure of steel components subjected to arbitrarily applied cyclic loading (such as that imposed by earthquakes) can be reliably estimated by decomposing the force displacement curve experienced by the metallic damper in the so-called skeleton and Bauschinger parts through an index of damage (ID). The skeleton part is formed by sequentially connecting the segments of the axial force-axial deformation curve of the damper that exceed the load level attained by the preceding cycle in the same domain of loading. The remaining part of the curve is called the Bauschinger part. The ID depends on the plastic strain energy dissipated in the skeleton and in the Bauschinger parts.

However, measuring the force and the displacement of the hysteretic damper during an earthquake entails installing expensive instrumentation (load cells or strain gages, displacement transducers, etc.) on the dampers. This reduces one of the main advantages of hysteretic dampers, low cost, and would not be justified for a type of action, earthquake, whose probability of occurrence is very low. Alternatively, the use of piezoceramic sensors permanently attached to the hysteretic damper provides a preferable (cheaper) solution for instrumenting the EDDs and allows for conducting simple vibration tests to evaluate the level of damage using structural health monitoring (SHM) strategies. Piezoceramic sensors are low-cost devices that can be used both to excite the dampers and to measure their response.

Recent research efforts in SHM technologies have been applied mainly to monitor bridges ${ }^{11} 16$ or structural elements such as beams or columns. ${ }^{17} 20$ However, few publications describe SHM technologies 
in the context of seismic control systems such as dampers. Research in this area involves numerical simulations of the response of a building model equipped with dampers and excited by means of dynamic signals, ${ }^{4,5}$ whereas few studies compare the numerical simulation results with real experimental outcomes.

Vibration-based damage detection strategies are based on the correlation between the vibration response of the structure and the presence of damage. ${ }^{21,22}$ Damage alters the vibration response of the structure (resonance frequencies, modes, damping coefficients, etc.), and this can be detected and evaluated by adequate processing of signals captured by the sensors and correlated with the presence (Level 1 in SHM), position (Level 2 in SHM), and intensity of damage (Level 3 in SHM).

A number of research studies deal with time vibration series analysis for damage detection in structures and materials (such as buildings, airplanes, and composites). ${ }^{21}{ }^{23}$ Most of these algorithms use natural frequencies as damage indicators. The principal advantages of frequency measurements are that signals can be quickly acquired ${ }^{24}$ and that brief damage detection methods can be implemented. Some of these methods are based on frequency response functions (FRFs). FRF-based methods are feasible indicators of structural damage and can obtain damage information from in situ measurements without an exhaustive modal analysis. FRF methods are straightforward ${ }^{25}$ and do not require many sensors to provide reliable information about the structural dynamic behaviour. ${ }^{26}$ Also, novel studies on structural diagnosis combine FRF with soft computing techniques ${ }^{27} 29$ to obtain enhanced results.

Research on the FRF-based method for damage detection may use direct measurement of FRF, ${ }^{30} \mathrm{FRF}$ curvatures, ${ }^{31}$ FRF differences, ${ }^{28}$ or compressed FRFs, ${ }^{27}$ but thus far, none of these techniques has been applied to evaluate the level of damage on hysteretic dampers through comparison or correlation with mechanical indexes of damage.

This article presents an experimental investigation on a practical SHM system proposed for a particular type of hysteretic EDD that consists of short segments of I-shaped steel sections whose source of energy dissipation is the plastification of the web. Four I-section steel segments were instrumented with piezoceramic sensors and subjected to the same pattern of cyclic loading. The applied force and the corresponding displacement were continuously recorded. At several stages of the cyclic loading, corresponding to different levels of damage referred to as $D_{i}$ hereafter, the cyclic loading was stopped, and the I-section steel segments were subjected to controlled white noise random vibrations. In these vibration tests, the web of the I-section was excited with a piezoceramic actuator attached to one side of the web, and the signal was recorded by a piezoceramic sensor attached to the opposite side. The loaddisplacement curve recorded up to the point where the cyclic loading stopped was decomposed to calculate an energy-based ID. Also, the signals recorded with the vibration tests conducted after each interruption of the cyclic loading were analyzed using a nonparametric FRF-based method. ${ }^{21}$ From these analyses, a new ID called area index damage (AID) was defined. A good correlation is found between ID and AID, and this indicates that the level of damage on the hysteretic EDD can be readily estimated from simple vibration tests, with no need to know the load displacement relationship and without resorting to cumbersome and expensive instrumentation.

\section{Description of hysteretic EDDs}

The hysteretic EDD investigated in this study consists of I-section steel segments that are assembled and installed in the structural frame in such a way that the energy is dissipated through out-of-plane plastic strains on the web of the I-section when the frame is subjected to lateral seismic loads. ${ }^{32}$ Figure 2 shows the typical Isection steel segment that constitutes the hysteretic EDD itself. Figure 3 shows its deformation pattern, the shaded region indicates the locations where plastic deformations occur. Figure 4 shows a simple solution for assembling the EDDs to form a structural member that can be installed in a frame as a conventional brace. This assemblage uses auxiliary steel elements called Usections that are designed to remain elastic, while the Isection steel segments undergo plastic deformations. The details on the dimension of U-sections are provided in Figure 4.

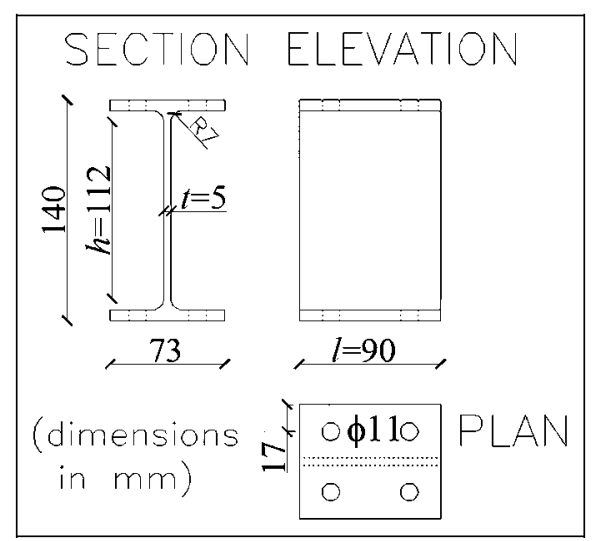

Figure 2. Hysteretic EDD: nominal dimensions of test specimens. 


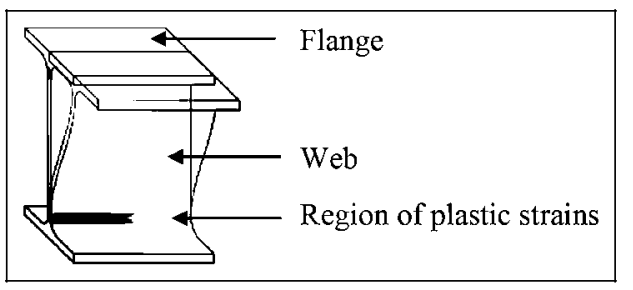

Figure 3. Deformation pattern of the EDD.

\section{Experimental investigation}

\section{Test specimens and experimental setup}

From a hot-rolled I-section steel profile, four short segments of identical length were cut to constitute the EDDs (test specimens) investigated in this study. They will hereafter be referred to as specimens I1 through I4. The nominal geometry of each specimen is shown in Figure 2. The specimens were mounted in the setup shown in Figure 5. The flanges of the test specimen were fastened to two U-shaped sections by posttensioning four high-strength bolts with a torque of 67 $\mathrm{Nm}$ each. One of the U-sections was securely fastened to a stiff reaction wall, while the other was connected through a pin-joint to an MTS $250 \mathrm{kN}$ capacity dynamic actuator. The centerline of the actuator was contained in the horizontal plane of symmetry of the test specimen. The piston of the actuator was allowed to displace in the horizontal direction. A counterweight was installed to avoid the self-weight of the testing apparatus from loading the test specimen. A load cell installed between the actuator and the pin-joint measured the horizontal restoring force $V$ opposed by the specimen when subjected to forced cyclic displacements. A displacement transducer connecting the upper flange of the I-section and the reaction wall provided the relative horizontal displacement $\delta$ between the upper and lower flanges of the test specimen.

\section{Cyclic tests: instrumentation and loading history}

Horizontal relative displacements were imposed with the actuator between the flanges of the I-section steel segments using the setup shown in Figure 5. The loading pattern consisted of successive cycles of increasing displacement amplitude, as shown in Figure 6, following the sequence $5 \delta_{y}, 10 \delta_{y}, 15 \delta_{y}, 20 \delta_{y}, 25 \delta_{y}$, and $30 \delta_{y}$. Here, $\delta_{y}(=1.5 \mathrm{~mm})$ is the horizontal yield displacement of the specimen ${ }^{32}$ calculated with equation (1)

$$
\delta_{y}=\frac{f_{y} h^{2}}{2 t E}
$$

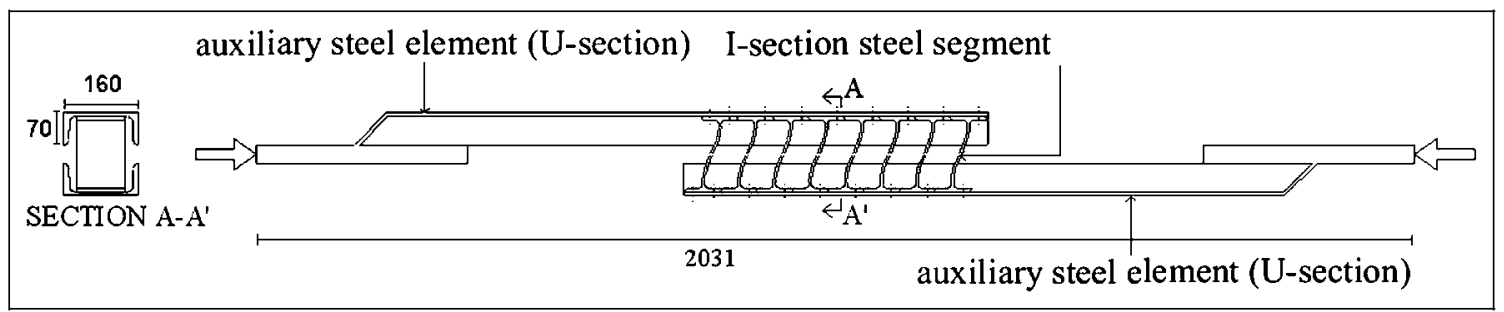

Figure 4. Assemblage of EDDs to form a brace-type structural member showing the main parts: I-section and U-section (dimensions in millimeter).

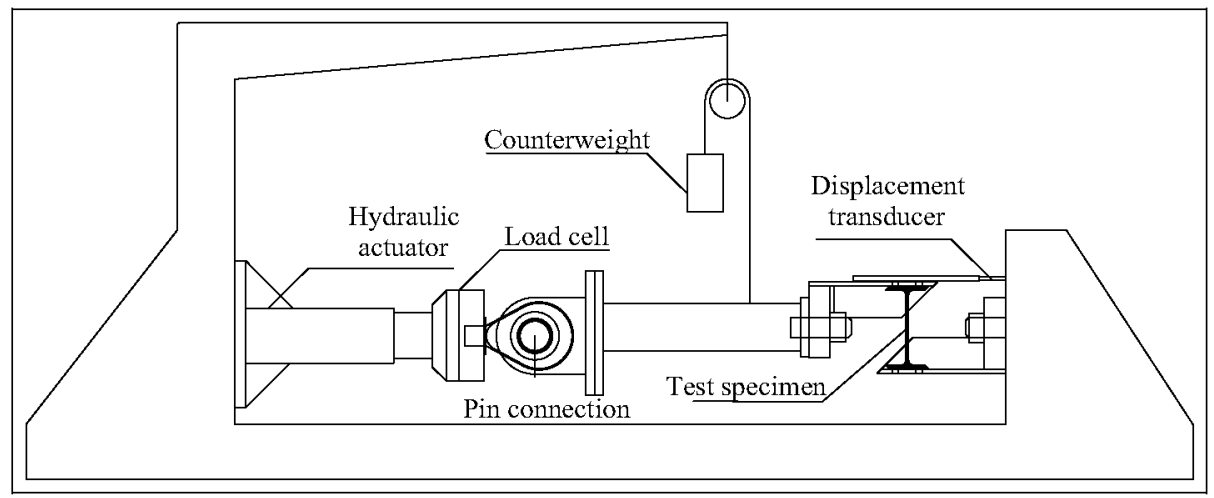

Figure 5. Setup of the experiment in the cyclic test. 


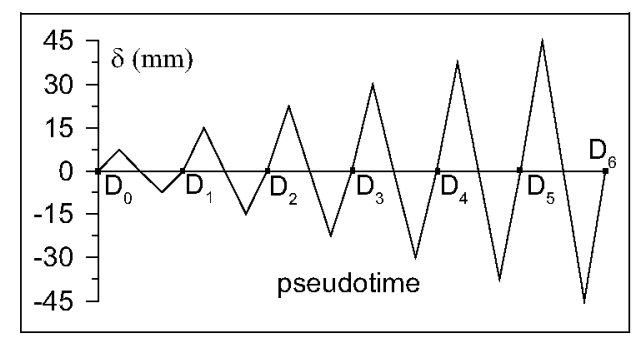

Figure 6. Loading history.

where $f_{y}$ is the yield stress of the steel, $E$ is its modulus of elasticity, and $h$ and $t$ are the height and web thickness of the I-section steel segment, respectively, as shown in Figure 2. The corresponding yield strength $V_{y}$ of the EDDs can also be estimated as follows ${ }^{32}$

$$
V_{y}=\frac{l t^{2} f_{y}}{2 h}
$$

Here, the new parameter $l$ is the length of the steel segment as shown in Figure 2. The cyclic rate applied by the actuator was $1 / 80 \mathrm{~Hz}$. The qualitative level of damage attained by a given specimen after $i$ cycles of loading is referred to as $D_{i}$ in the horizontal axis of Figure 6. $D_{0}$ represents the initial undamaged state. Specimen I1 was subjected to five cycles and specimens I2, I3, and I4 to six cycles of displacement.

\section{Vibration tests: instrumentation, applied signal, and preprocessing}

Before applying the cyclic loading on the test specimen, that is, when the level of damage was $D_{0}$, and at intermediate and/or final levels of damage $D_{i}$, the specimens were subjected to vibration tests. For specimens I1 and $\mathrm{I} 2$, vibration tests were conducted only at the end of the cyclic test, while for specimens I 3 and I4, vibration tests were carried out at the end of each cycle to investigate the progress of damage. To conduct the vibration tests, the web of the specimen was instrumented with one piezoelectric ceramic (lead zirconate titanate (PZT)) sensor glued on one side and another PZT sensor glued on the opposite side at the location shown in Figure 7. The location of the PZTs was determined using the criterion of separating the PZT from the regions of largest plastic deformation in order to avoid damaging the PZT. In the EDDs under study, maximum plastic strain areas were expected at both ends of the web of the I-section (see Figure 3), while strains at the center of the web are nominally zero. Therefore, the PZTs were placed at the center of the web of the I-section.

The vibration tests consisted of exciting the web of the I-section by means of a controlled vibration signal $x[t]$ applied at the PZT on one side of the web

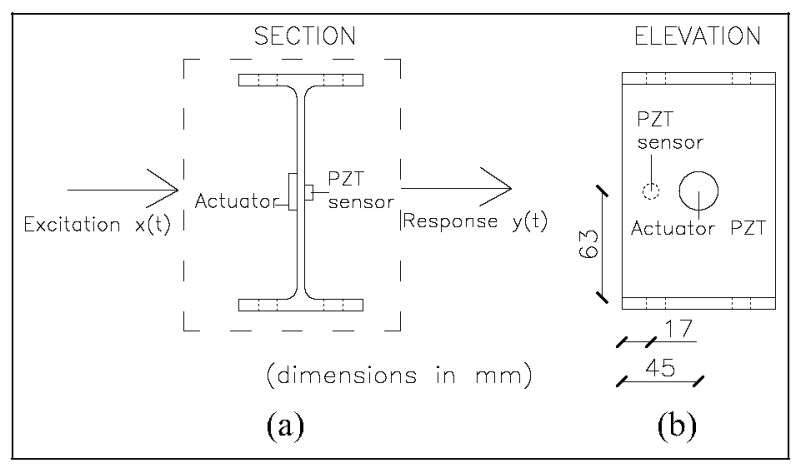

Figure 7. Position of sensors on specimen: (a) section view and (b) frontal view. PZT: lead zirconate titanate.

("actuator" in Figure 7) and recording simultaneously the response at the PZT located on the opposite side ("sensor" in Figure 7). The signal recorded by the sensor is referred to as $y[t]$ hereafter. The PZT used as actuator was a PI ${ }^{(\mathbb{B}} \mathrm{PRYY} 0842$. The PZT used as sensor was a PI ${ }^{\circledR} \mathrm{PRYY} 0220$. The signals $y[t]$ recorded with the PZT sensor had a voltage amplitude of about $3 \mathrm{Vp}$.

Brüel \& Kjær PULSE equipment was used as signal generator of the random excitation $x[t]$ and as acquisition system to record the response vibration signals $y[t]$. The PULSE system provides an output signal with a maximum peak at about $5 \mathrm{~V}$, which was seen to be insufficient to properly excite the specimen. Therefore, a gain $G=20$ was used. In addition, to ensure good quality of the signals, the acquisition system was configured in the differential mode. This configuration made it possible to measure the signal of each PZT as the difference between the two channels, thus avoiding any reference to the electric ground. Moreover, to avoid both mechanical and electric noise, a ground system was installed throughout the cable network and connected to an embedded mass system that was independent of the ground system of the laboratory building.

For both $x[t]$ and $y[t]$ signals, the sampling frequency used was $f_{s}=65,536 \mathrm{~Hz}$, which corresponds to a sampling period of $\Delta t=1.5 \times 10^{-5} \mathrm{~s}$. The total number $N$ of data recorded was $N=657,408$, corresponding to 10 $s$ of acquisition time. For statistical reasons, five vibration tests were carried out for each level of damage $D_{i}$ and for each specimen, using a different white noise sequence in each but maintaining the same frequency content and variance. The signals recorded during the vibration tests were suitably preprocessed for data conditioning purposes. This preprocessing consisted of the following steps:

1. Filtering. A band-pass filter of bandwidth (BW) of $0.3-25 \mathrm{kHz}$ was applied to prevent electrical noise 
Table I. Eigenfrequencies $(\mathrm{kHz})$ of the undamaged specimens.

\begin{tabular}{lrrrr}
\hline Test specimen & \multicolumn{1}{l}{ II } & \multicolumn{1}{c}{12} & \multicolumn{1}{l}{13} & \multicolumn{1}{l}{14} \\
\hline Mode 1 & 1.56 & 1.58 & 1.59 & 1.57 \\
Mode 2 & 4.57 & 4.59 & 4.57 & 4.59 \\
Mode 3 & 9.56 & 9.58 & 9.50 & 9.57 \\
Mode 4 & 23.93 & 24.04 & 23.91 & 23.95 \\
\hline
\end{tabular}

and avoid nonlinear performance of the sensors. Finite element numerical simulations conducted prior to the tests confirmed that at least 17 eigenfrequencies of the test specimen were located inside the frequency BW of $0.3-25 \mathrm{kHz}$. This BW was considered sufficient for damage detection and evaluation purposes. It is worth noting that although the four specimens were cut from the same profile, due to the hot rolling process, tolerances up to about $\pm 10 \%$ from the nominal size can be expected. Because of these variations on the nominal geometry, the eigenfrequencies of the four specimens before applying any damage were slightly different. Table 1 shows the values of four relevant eigenfrequencies used in this study for health monitoring purposes, as explained later.

2. Normalization and scaling. Due to numerical reasons, in order to counteract different excitation levels and environmental conditions and in order to compensate the offset and amplitude variability among the signals, the following normalization process was applied

$$
x[t]_{n}=\frac{x[t]-\bar{x}[t]}{\sigma(x)} ; y[t]_{n}=\frac{y[t]-\bar{y}[t]}{\sigma(y)}
$$

where $x[t]_{n}$ and $y[t]_{n}$ are the normalized signals, $\bar{x}[t]$ and $\bar{y}[t]$ are their mean values, and $\sigma(x)$ and $\sigma(y)$ are their standard deviation, respectively. For the sake of clarity, from now on, the sub-index $n$ will be removed and the simpler notations $x[t]$ and $y[t]$ will be used for the normalized signals instead of $x[t]_{n}$ and $y[t]_{n}$, that is, $x[t]$ $\equiv x[t]_{n}$ and $y[t] \equiv y[t]_{n}$. Thus, after acquisition and signal conditioning, vibration data recorded for each specimen were collected in a vector denoted as $z[t]=[x[t]$, $y[t]]^{T}$.

\section{Mechanical damage evaluation from the $V-\delta$ curves through index $I D_{i}$}

\section{Decomposition of the $V-\delta$ curves}

Figure 8(a) shows the typical load-displacement relationship, $V-\delta$, obtained from the cyclic tests. The $V-\delta$ curve exhibited by the test specimen up to a given level

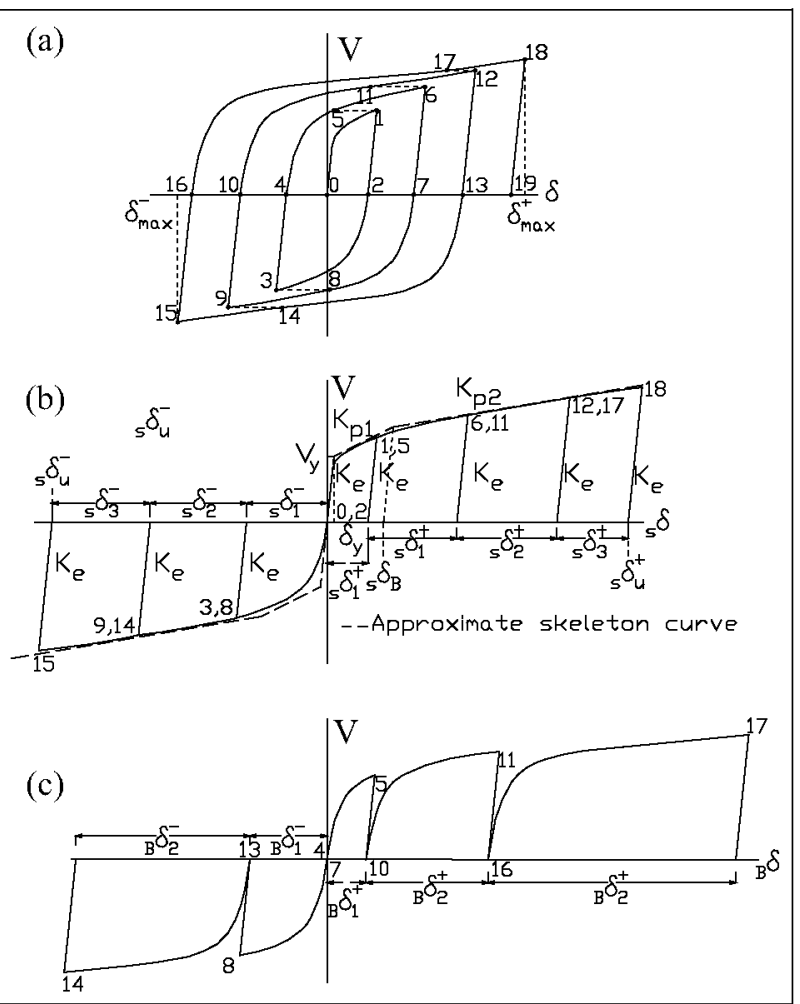

Figure 8. Decomposition of a typical $V-\delta$ curve: (a) initial $V-\delta$ curve, (b) skeleton part, and (c) Bauschinger part.

of damage $D_{i}$ defined by point $i$ of the coordinates $\left(V_{i}-\delta_{i}\right)$ can be decomposed into the so-called skeleton part and the Bauschinger part as shown in Figure 8(b) and (c), respectively. The skeleton part (Figure $8(\mathrm{~b})$ ) is derived by sequentially connecting the segments $0-1,5$ $6,11-12$, and $17-18$ in the positive domain and $2-3,8$ 9 , and $14-15$ in the negative domain - these being the paths that exceed the load level attained by the preceding cycle in the same domain of loading. Segments $1-2$, 6-7, 12-13, 18-19, 3-4, 9-10, and 15-16 are the unloading paths, whose slope coincides with the initial elastic stiffness $K_{e}\left(=V_{y} / \delta_{y}\right)$. For each domain of loading, the area enveloped by the skeleton curve up to a given point $\left(V_{i}, \delta_{i}\right)$ will be called ${ }_{s} W_{i}^{+}$and ${ }_{s} W_{i}^{-}$. The segments $4-5$, 10-11, and 16-17 in the positive domain and 7-8 and 13-14 in the negative domain of loading - which begins at $V=0$ and terminates at the maximum load level previously attained in preceding cycles in the same loading domain - are the so-called Bauschinger part (Figure 8(c)). For each domain of loading, the sum of the areas enveloped by the Bauschinger part up to a given point $\left(V_{i}, \delta_{i}\right)$ will be referred to as ${ }_{B} W_{i}^{+}$and ${ }_{B} W_{i}^{-}$. The sum ${ }_{s} W_{i}^{+}+{ }_{B} W_{i}^{+}$in the positive domain, and ${ }_{s} W_{i}^{-}+{ }_{B} W_{i}^{-}$in the negative domain, gives the total plastic strain energy dissipated by the EDD in each domain of loading. For convenience, these energies can be expressed in 
Table 2. Value of the index of damage.

\begin{tabular}{lllll}
\hline Specimen & 11 & 12 & 13 & 14 \\
\hline$D_{1}$ & & & 0.02 & 0.02 \\
$D_{2}$ & & 0.08 & 0.08 \\
$D_{3}$ & & 0.18 & 0.22 \\
$D_{4}$ & & & 0.33 & 0.41 \\
$D_{5}$ & 0.54 & & 0.51 & 0.62 \\
$D_{6}$ & & 0.72 & 0.67 & 0.81 \\
\hline
\end{tabular}

nondimensional form in terms of the new ratios ${ }_{s} \bar{\eta}_{i}^{+}$, $\bar{\eta}_{i}^{+},{ }_{s} \bar{\eta}_{i}^{-}$, and $\bar{\eta}_{i}^{-}$defined as follows

$$
\begin{aligned}
& { }_{s} \bar{\eta}_{i}^{+}=\frac{{ }_{s} W_{i}^{+}}{V_{y} \delta_{y}} ; \overline{\boldsymbol{\eta}}_{i}^{+}=\frac{{ }_{s} W_{i}^{+}+{ }_{B} W_{i}^{+}}{V_{y} \boldsymbol{\delta}_{y}} ; \\
& s \bar{\eta}_{i}^{-}=\frac{{ }_{S} W_{i}^{-}}{V_{y} \delta_{y}} ; \overline{\boldsymbol{\eta}}_{i}^{-}=\frac{{ } W_{i}^{-}+{ }_{B} W_{i}^{-}}{V_{y} \boldsymbol{\delta}_{y}}
\end{aligned}
$$

\section{Evaluation of mechanical damage through an energy- based index ID}

Past research ${ }^{10}$ showed that the level of mechanical damage in a metallic EDD subjected to arbitrarily applied cyclic loading up to a point $i\left(V_{i}, \delta_{i}\right)$ can be accurately predicted using the following index

$$
\mathrm{ID}_{i}=\max \left\{\overline{\mathrm{ID}}_{i}^{+}, \overline{\mathrm{ID}}_{i}^{-}\right\}
$$

where

$$
\overline{\mathrm{ID}}_{i}^{+}=\frac{\bar{\eta}_{i}^{+}}{\bar{\eta}_{u}^{+}}, \overline{\mathrm{ID}}_{\mathrm{i}}^{-}=\frac{\bar{\eta}_{i}^{-}}{\bar{\eta}_{u}^{-}}
$$

In equation (6), $\bar{\eta}_{u}^{+}$and $\bar{\eta}_{u}^{-}$represent the ultimate energy dissipation capacity of the EDD, which depends on the values of ${ }_{s} \bar{\eta}_{i}^{+}, \bar{\eta}_{i}^{+},{ }_{s} \bar{\eta}_{i}^{-}$, and $\bar{\eta}_{i}^{-}$and on the two empirical parameters $a$ and $b$ related to the material properties of the steel and the geometry of the EDD. A detailed explanation of these parameters and their value for the type of EDD investigated in this study can be found in Benavent-Climent et al. ${ }^{32}$ The value $I D_{i}=0$ indicates no damage, while $I D_{i}=1$ means complete failure. The values of the mechanical index of damage $I D_{i}$ calculated for the damage levels $D_{1}, D_{2}$, $D_{3}, D_{4}, D_{5}$, and $D_{6}$ according to Benavent-Climent et al. $^{32}$ are summarized in Table 2 .

\section{Damage assessment by processing vibration test results: AID index}

Level 1 in SHM is used to ascertain whether the inspected specimen, named $S_{u}$ hereafter, has damage or not. At this stage, a decision must be made between two options: hypothesis $H_{o}$, under which the inspected specimen is considered to be undamaged $\left(S_{o}\right)$ and the opposite hypothesis, $H_{1}$, under which the inspected specimen is considered to be damaged. Such conditions are mathematically expressed as follows ${ }^{21}$

$$
H_{o}: S_{u}=S_{o}: \text { null hypothesis (undamaged specimen) }
$$

$H_{1}: S_{u} \neq S_{o}:$ alternative hypothesis (damaged specimen)

The decision between hypotheses $H_{o}$ and $H_{1}$ is taken on the basis of a quantitative parameter called characteristic quantity $Q$ along with some statistical considerations.

The statistical time series method for damage detection entails three phases. ${ }^{21}$ The first phase, called the baseline phase, consists of data acquisition and statistical modeling of the undamaged specimen $S_{o}$; in this phase, from the vibration response data, $z_{o}[t]$, the estimation of characteristic quantity $Q_{o}$ is made. In the second phase, the inspection phase, the characteristic quantity $Q_{u}$ is estimated from the vibration response data, $z_{u}[t]$, of the inspected structure $S_{u}$. Finally, the statistical decision-making phase (equations (7) and (8)) consists of comparing $Q_{u}$ and $Q_{o}$ by means of statistical tests. A general scheme is offered in Figure 9.

\section{FRF-based method for damage detection and statistical test}

Past research ${ }^{33}$ showed that the FRF is a good method to detect the presence of damage on the EDD investigated; it is used in this study as the characteristic quantity $Q$. The FRF-based method uses both excitation $x[t]$ and response signals $y[t]$, and its physical basis is the detection of changes in the frequency response of the specimen when damage appears, that is, damage detection is based on confirmation of statistically significant deviations (from the healthy $S_{o}$ ) in the inspected specimen FRF at some frequencies. ${ }^{21}$

The magnitude of FRF, $|\hat{H}(j \omega)|$, is calculated as

$$
Q_{F R F}=|\hat{H}(j \omega)|=\frac{\left|\hat{S}_{x y}(j \omega)\right|}{\hat{S}_{x}(\omega)}
$$

where $\left|\hat{S}_{x y}(j \omega)\right|$ and $\left|\hat{S}_{x}(\omega)\right|$ are the cross and autospectral density functions, respectively, estimated via the Welch method. ${ }^{34}$

This estimator of the FRF, $|\hat{H}(j \omega)|$, has an approximately normal distribution with mean the true FRF, $|H(j \omega)|$, and variance, $\sigma^{2}(\omega)$, as is demonstrated in Bendat and Piersol, ${ }^{35}$ that is

$$
|\hat{H}(j \omega)| \sim N\left(|H(j \omega)|, \sigma^{2}(\omega)\right)
$$

where the variance is given by 


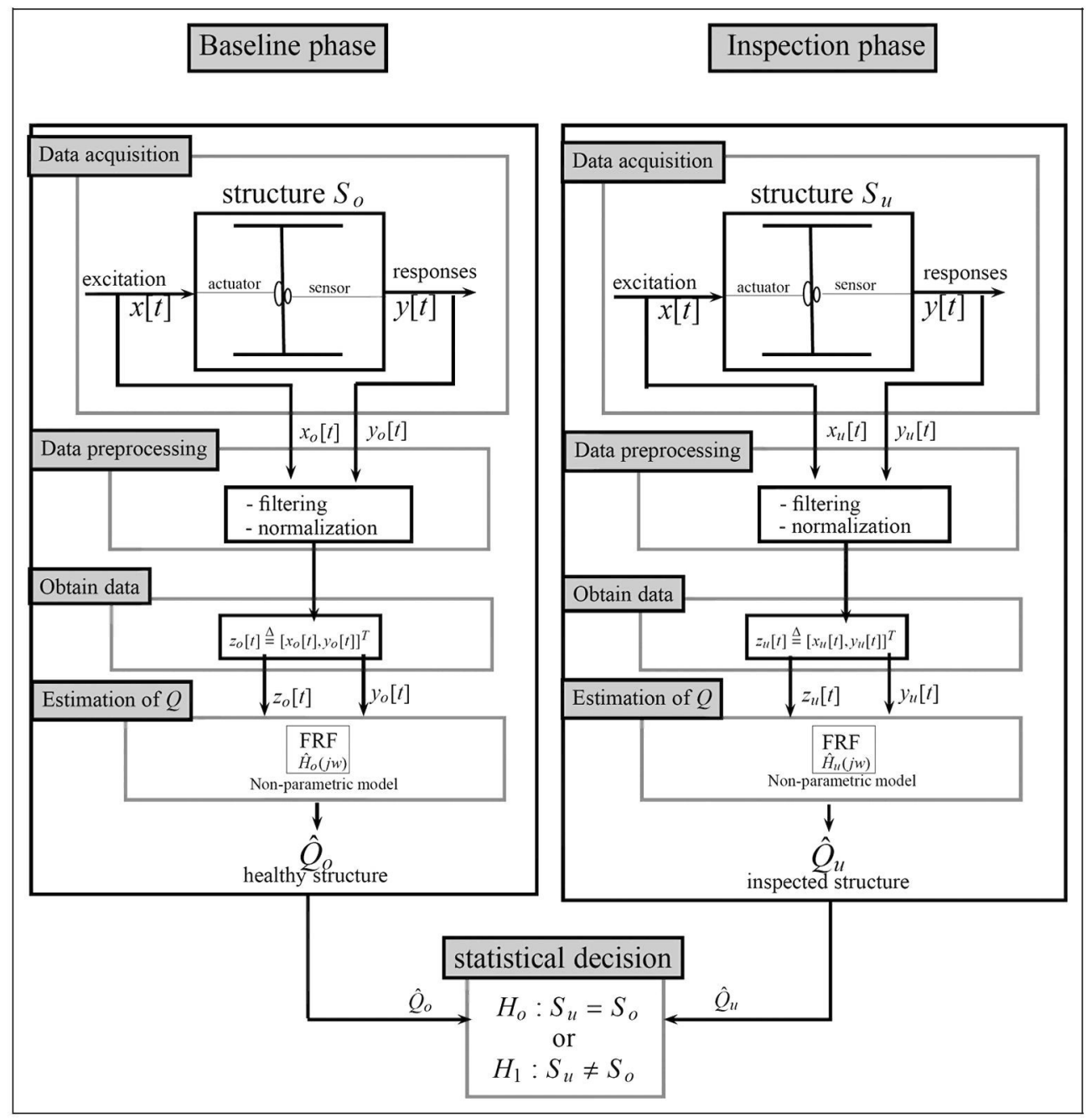

Figure 9. General scheme of the statistical time series methods for damage detection.

FRF: frequency response function.

$$
\sigma^{2}(\omega) \approx \frac{1-\gamma^{2}(\omega)}{\gamma^{2}(\omega) 2 K}|H(j \omega)|^{2}
$$

$K$ being the number of segments in which the used data are divided, and

$$
\gamma^{2}(\omega)=\frac{\left|S_{x y}(j \omega)\right|^{2}}{S_{x}(\omega) S_{y}(\omega)}
$$

the coherence function. ${ }^{21}$

Thus, the algorithm to detect the damage based on the FRF estimator can be summarized in the follow steps. $^{21,33}$

1. Obtain the characteristic quantity $Q_{0}$ for the healthy structure: this quantity is calculated following equation (9) using the available data $z_{o}[t]$ to estimate the magnitude $\left|\hat{H}_{o}(j \omega)\right|$.
2. Obtain the characteristic quantity $Q_{u}$ for the inspection structure: in the same way, $Q_{u}$ is calculated with equation (9) using the available data $z_{u}[t]$ to estimate the magnitude $\left|\hat{H}_{u}(j \omega)\right|$.

3. Set up the statistical hypothesis-testing problem for fault detection: the hypothesis test is configured using the characteristic quantity selected and following equations (7) and (8). In this case, the hypothesis test is formulated as

$$
\begin{aligned}
& H_{o}:\left|H_{o}(j \omega)\right|-\left|H_{u}(j \omega)\right|=0 \\
& H_{1}:\left|H_{o}(j \omega)\right|-\left|H_{u}(j \omega)\right| \neq 0
\end{aligned}
$$

4. Establish the statistics: for this case, the statistic is expressed as the difference between the magnitudes estimated for healthy and damaged structures 


$$
\delta|\hat{H}(j \omega)|=\left|\hat{H}_{o}(j \omega)\right|-\left|\hat{H}_{u}(j \omega)\right|
$$

Note that since $\left|\hat{H}_{o}(j \omega)\right|$ and $\left|\hat{H}_{u}(j \omega)\right|$ are independent normal variables, the difference has a distribution with mean $\delta|H(j \omega)|$ and variance $\delta \sigma^{2}(\omega)$, that is

$$
\delta|\hat{H}(j \omega)| \sim N\left(\delta|H(j \omega)|, \delta \sigma^{2}(\omega)\right)
$$

where $\delta|H(j \omega)|=\left|H_{o}(j \omega)\right|-\left|H_{u}(j \omega)\right|$ and $\delta \sigma^{2}(\omega)=\sigma_{o}^{2}$ $(\omega)+\sigma_{u}^{2}(\omega)$.

5. Evaluate the statistic under the null hypothesis: under $H_{o}$ (equation (13)), it is assumed that the inspection specimen is healthy, the two real magnitudes are the same $\left|H_{o}(j \omega)\right|=\left|H_{u}(j \omega)\right|$, and the variances are the same as $\sigma_{o}^{2}(\omega)=\sigma_{u}^{2}(\omega)$. Then, the distribution of $\delta|\hat{H}(j \omega)|$ (equation (15)) can be written as

$$
\delta|\hat{H}(j \omega)| \sim N\left(0,2 \sigma_{o}{ }^{2}(\omega)\right)
$$

6. For convenience, the statistic is normalized to have a standard normal distribution, that is

$$
Z=\frac{\delta|\hat{H}(j \omega)|}{\sqrt{2 \hat{\sigma}_{0}^{2}(\omega)}} \sim N(0,1)
$$

where $\hat{\sigma}^{2}{ }_{o}(\omega)$ is the variance of FRF estimated for the undamaged specimen in the baseline phase. ${ }^{21}$

As long as $Z_{\alpha / 2} \leqslant Z \leqslant Z_{1-\alpha / 2}$, that is, the $Z$-statistic remains within the confidence interval, the null hypothesis $H_{o}$ will be accepted and the inspected specimen will be considered undamaged. Otherwise, if the inequation is not satisfied, the hypothesis $H_{1}$ will be accepted and the specimen will be considered as damaged. $\alpha$ determines the probability of false alarm and is called risk level, whereas $Z_{\alpha / 2}$ and $Z_{1-\alpha / 2}$ are called the critical points. ${ }^{21,36}$

\section{Damage estimation: definition of damage index AID}

In Figure 10, the $|\mathrm{Z}|$-statistic is plotted against the frequency. If the entire $|Z|$-statistic plot is under the critical point (dashed horizontal line at level $|Z|=Z_{1-\alpha / 2}$ ) in Figure 10(a), the inspected specimen is assumed to be undamaged with the level of certainty associated with $\alpha$. Otherwise, if some peaks of the $|Z|$-statistic cross the critical point (Figure 10(b)), the inspected specimen is considered damaged. ${ }^{36}$

Previous experiments ${ }^{33}$ on the type of EDD investigated showed that when the magnitude of damage increases, the area of $|Z|$-statistic increases proportionally in some frequencies sensitive to damage. Thus, an $A I D$ was proposed to estimate the intensity of the damage (Level 3 in SHM) following this equation

$$
A I D=\sum_{\omega_{\min }}^{\omega_{\max }}|Z(\omega)|, \quad \forall Z(\omega) \geq Z_{1-\alpha / 2}
$$

\section{Analysis of signals obtained from vibration tests and calculation of AID}

Figure 11 shows the magnitude of FRF spectra estimated from vibration response signals $y[t]$ of one of the specimens (I4) for each level of damage, that is, from $D_{0}$ to $D_{6}$, in the whole BW of [0.3-25] kHz (for clarity, the full BW has been divided into five separate graphs of $5 \mathrm{kHz}$ wide).

The following parameters to estimate the Welchbased FRF were used: (1) 128,000 sample windows; (2) number segments, $K=5$, without overlapping; and (3) frequency resolution of the FRF estimated $=0.512 \mathrm{~Hz}$. In Figure 11, four regions of the spectra, namely, BW1,

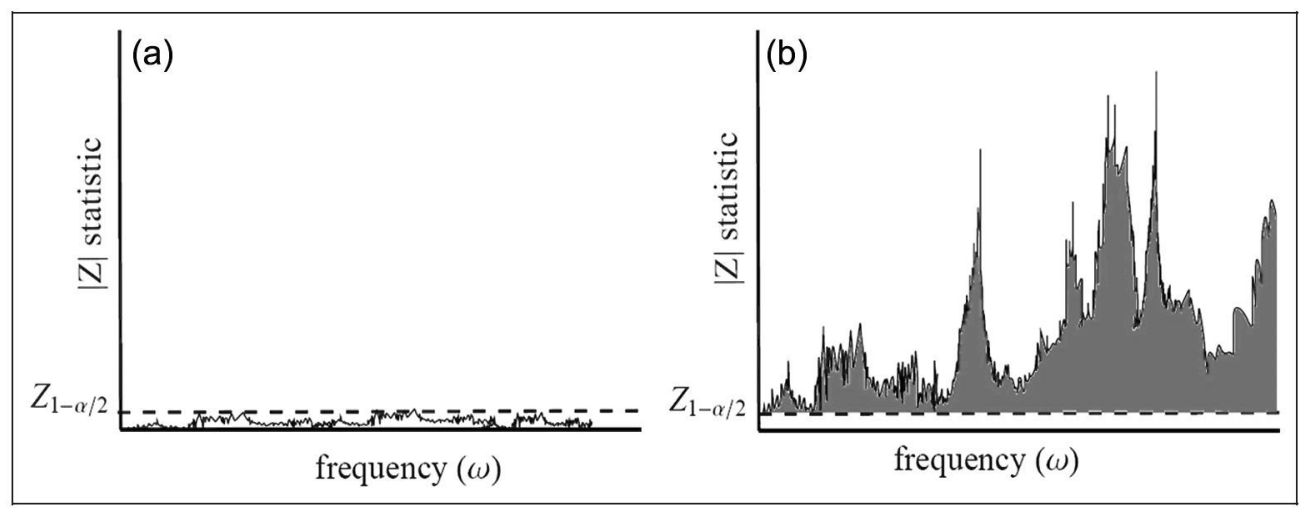

Figure 10. Graphic representation of the Z-statistic for (a) an undamaged specimen and (b) a damaged specimen. 


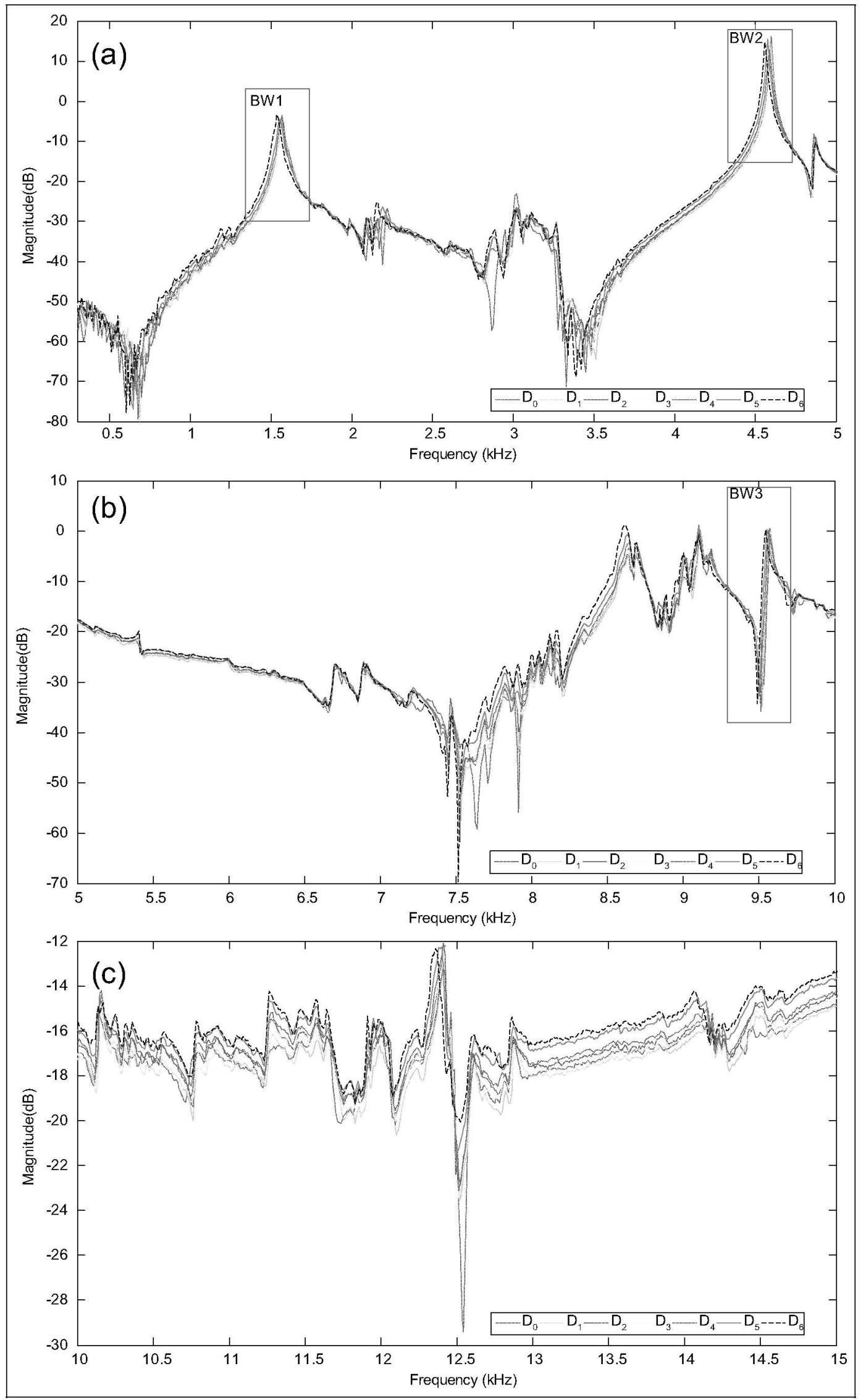

Figure II . (continued) 

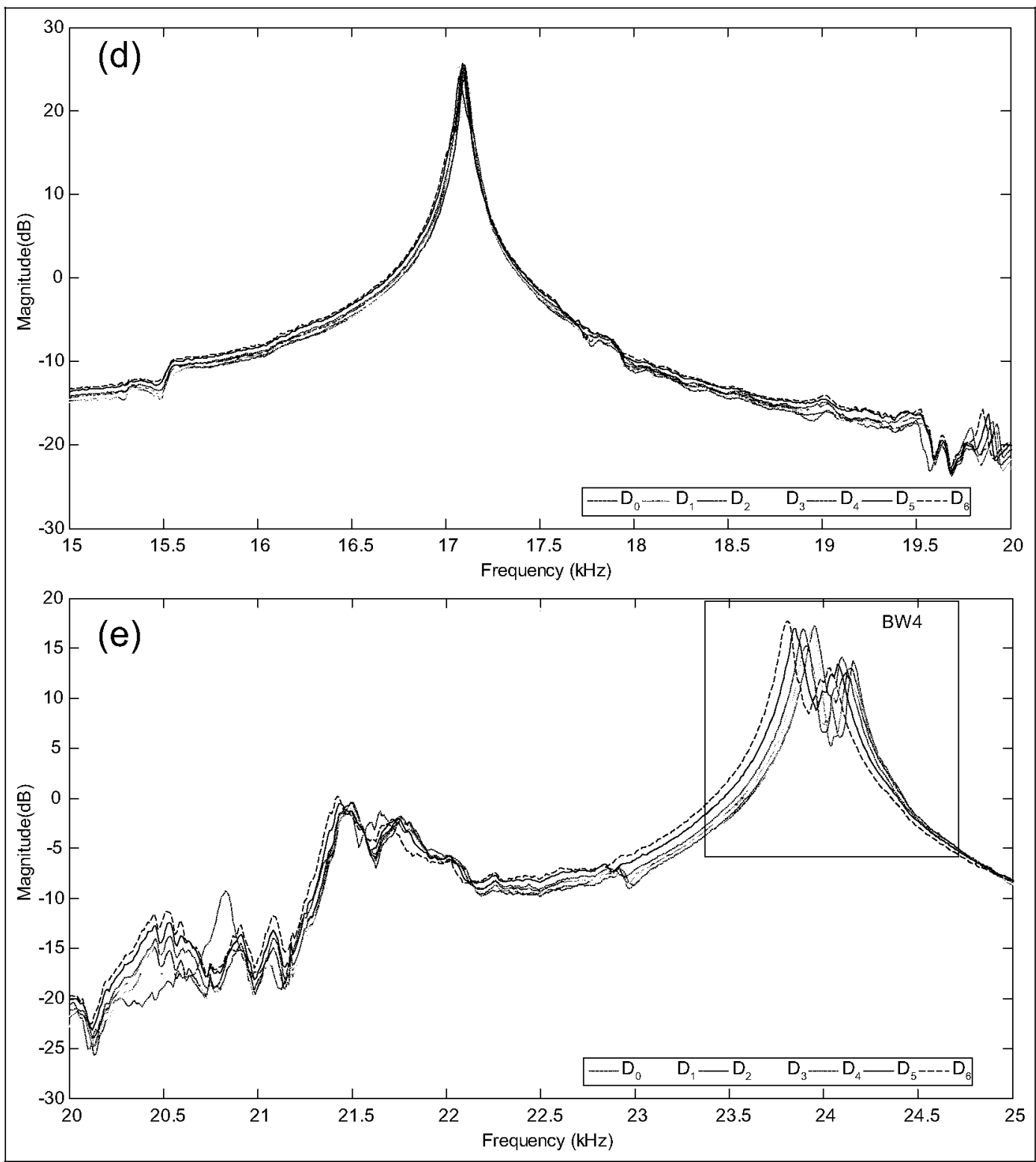

Figure I I. Magnitude of FRF spectra estimated for specimen 14 on bandwidth (a) [0-5], (b) [5-10], (c) [10-15], (d) [15-20], and (e) [20-25] kHz for all levels of damage (bandwidths sensitive to damage are highlighted in rectangular box in (a), (b), and (e); no bandwidths sensitive to damage are selected in (c) and (d)). BW: bandwidth.

$\mathrm{BW} 2, \mathrm{BW} 3$, and BW4, which are considered more sensitive to damage, are given in the rectangular box. The selection of these regions was made on the basis of previous finite element calculations, which indicated that the shape of the vibration modes associated with these frequency ranges provided the largest displacements at the location of the PZTs.

The BWs of these enclosed areas are as follows: $\mathrm{BW} 1=[1.4-1.7], \mathrm{BW} 2=[4.4-4.7], \mathrm{BW} 3=[9.4-9.7]$, and BW4 $=[23.424 .7] \mathrm{kHz}$. Figure 12 shows zooms of these frequency BWs, where a clear shift of the frequency response can be observed, which clearly increases with the level of damage.

It can be seen that the total frequency shift from the undamaged structure, $D_{0}$, to damage level $D_{6}$ is only about $40 \mathrm{~Hz}$ in the first bands, BW1 and BW2, and it increases in high frequencies to about $100 \mathrm{~Hz}$ in BW3 and about $190 \mathrm{~Hz}$ in BW4. The statistical test presented in section "Damage assessment by processing vibration test results: AID index" was applied to each specimen and for each BW1-BW4. As an example, Figure 13 shows the statistical test results for the 


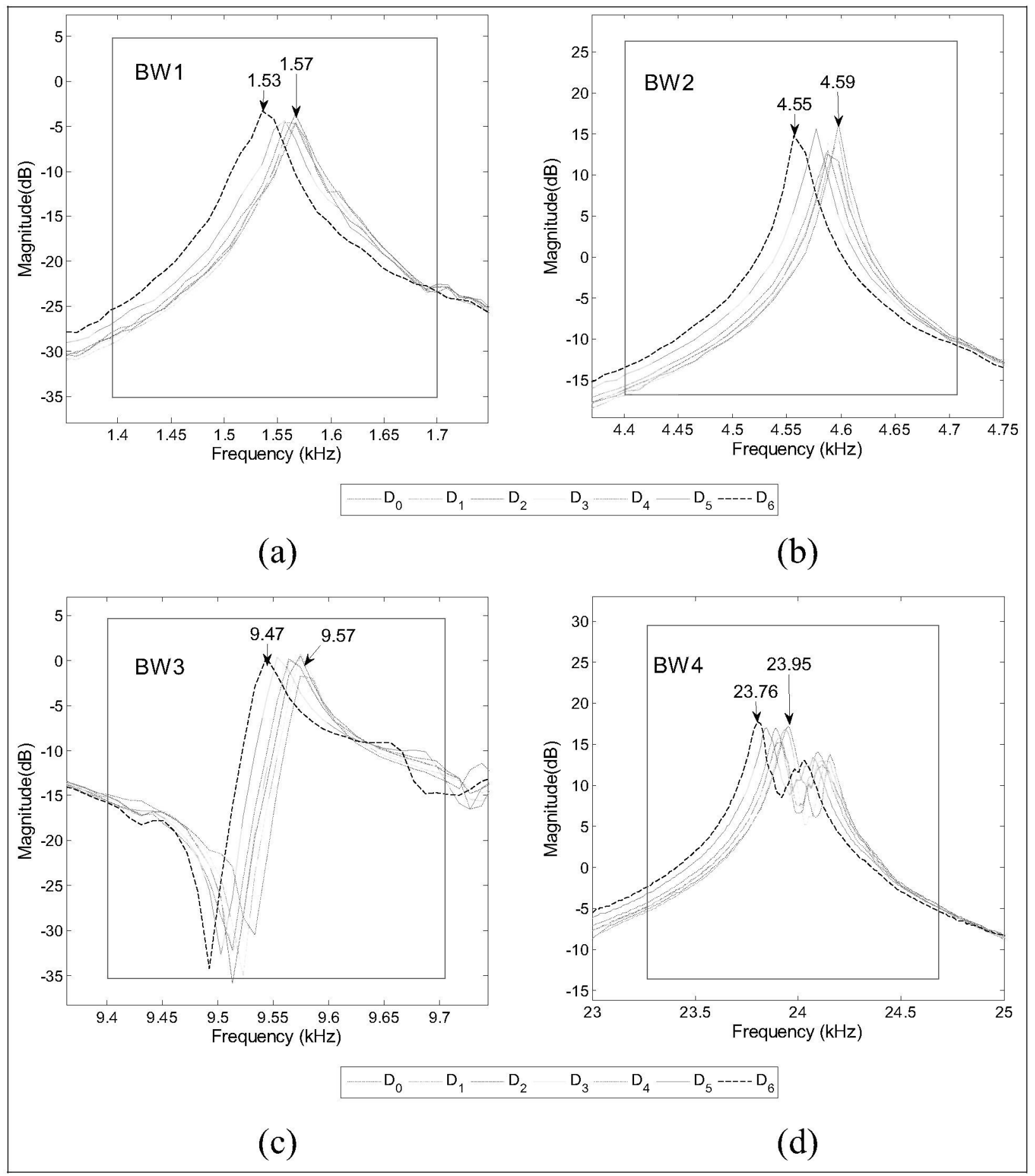

Figure I2. Details of four bandwidths sensitive to damage selected from the magnitude of FRF spectra estimated in specimen 14 for the levels of damage $D_{0}, D_{1}, D_{2}, D_{3}, D_{4}, D_{5}$, and $D_{6}$ : (a) $B W I=[1.4-1.7] \mathrm{kHz}$, (b) BW2 = [4.4-4.7] kHz, (c) BW3 = [9.4-9.7] $\mathrm{kHz}$, and (d) $\mathrm{BW} 4=[23.4-24.7] \mathrm{kHz}$.

BW: bandwidth.

frequency band $\mathrm{BW} 2=[4.4-4.7] \mathrm{kHz}$ for specimen I4. Typical false alarm probability with $\alpha=0.025$ on the standard normal density function was used. It can be clearly seen that the area of the statistical test, $Z$ - statistic, increases as the damage level increases (i.e. characterizing the index of damage AID defined in this section). The results did not show any false alarm cases. 

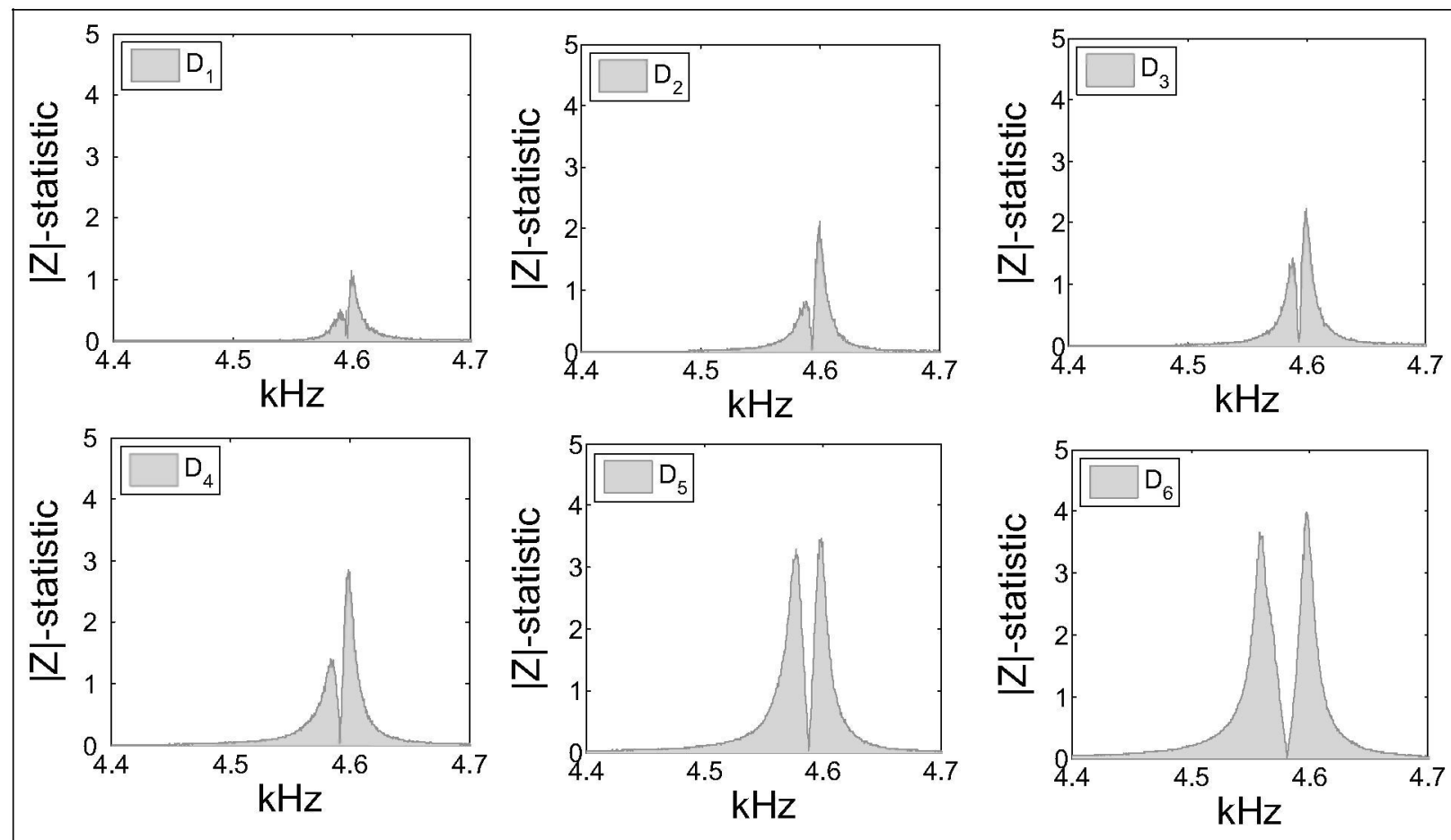

Figure 13. Application of the FRF-based statistical test to different levels of damage for the specimen 14 and $B W 2=[4.4-4.7] \mathrm{kHz}$.

For the four specimens tested, Table 3 summarizes the values of the index AID calculated in each BW and level of damage $D_{i}$. As seen in Table 3, the range of values of AID varies depending on the frequency band considered. The range is similar for BW1, BW2, and BW3 (between approximately 25 and 300) but increases notably for BW4 (between 230 and 1300). This means that the highest frequency band is most sensitive to damage. It is also worth noting that the values of index AID for the same level of damage $D_{i}$ (and especially for $D_{6}$ ) are similar among the four specimens, which indicates that the technique applied to the EDDs is robust.

In order to give an idea of how the group data from different tests are dispersed, Figure 14 shows an analysis of the dispersion of the AID for the specimen I4 and the four frequency bands and the six levels of damage used. The whiskers, in black color, show the maximum and minimum values obtained for AID in each test, and the boxes have lines at the median, lower, and upper quartile values. We can clearly appreciate the stability of the results against the random noise used as excitation of the healthy and inspected specimens. In general, the dispersion is small and has little influence on the results and its stability. This fact supports the validity and usefulness of the correlation given by equations (19) and (20) proposed in the next section.

\section{Correlation between the indexes ID and AID: prediction of ID from AID}

In Figure 15, the index AID is represented against the ID for the four frequency ranges investigated. It is clear from the figures that the relation between AID and ID for the four specimens in each frequency band can be approximated by a single curve/line. This means that the AID-ID relationship follows a clear and common pattern irrespective of the specimen considered.

Except in the lowest frequency range, in which the relation between AID and ID follows an approximately logarithmic law, in all the other frequency ranges, the relation between AID and ID is clearly linear and can be approximated by the following equations

Frequency ranges $[4.4-4.7]$ and $[9.4-9.7] \mathrm{kHz}$ :

$$
I D=0.0035 A I D=-0.25
$$

Frequency range $[23.4-24.7] \mathrm{kHz}$ :

$$
\mathrm{ID}=0.00075, \text { AID }=-0.20
$$

Comparing Figure 15(b) to (d), it is clear that the fit to a straight line improves as the frequency range increases. Among the four frequency ranges investigated, the band between 23.4 and $24.7 \mathrm{kHz}$ exhibits the highest sensitivity to damage, and the best fit of AID/ ID points to a straight line. These facts make the frequency band $23.4-24.7 \mathrm{kHz}$ as the preferable one to 
Table 3. Value of index AID.

\begin{tabular}{|c|c|c|c|c|c|}
\hline Bandwidth $(\mathrm{kHz})$ & Specimen damage level & II & 12 & 13 & 14 \\
\hline \multirow[t]{6}{*}{$\mathrm{BWI}=[1.4-1.7]$} & $D_{1}$ & & & 49.49 & 25.81 \\
\hline & $D_{2}$ & & & 48.72 & 36.05 \\
\hline & $D_{3}$ & & & 50.58 & 38.58 \\
\hline & $D_{4}$ & & & 63.34 & 71.47 \\
\hline & $D_{5}$ & 107.05 & & 116.05 & 150.17 \\
\hline & $D_{6}$ & & $28 \mid .83$ & 266.21 & 321.38 \\
\hline \multirow[t]{6}{*}{$\mathrm{BW} 2=[4.4-4.7]$} & $D_{1}$ & & & 62.69 & 34.13 \\
\hline & $D_{2}$ & & & 86.92 & 68.36 \\
\hline & $D_{3}$ & & & 109.86 & 85.09 \\
\hline & $D_{4}$ & & & 157.65 & 116.07 \\
\hline & $D_{5}$ & 155.15 & & 217.04 & 212.98 \\
\hline & $D_{6}$ & & 309.62 & 295.35 & 287.94 \\
\hline \multirow[t]{6}{*}{$\mathrm{BW} 3=[9.4-9.7]$} & $D_{1}^{\circ}$ & & & 118.70 & 72.37 \\
\hline & $D_{2}$ & & & 86.52 & 111.68 \\
\hline & $D_{3}$ & & & 128.89 & 126.75 \\
\hline & $D_{4}$ & & & 163.55 & 157.47 \\
\hline & $D_{5}$ & 189.32 & & 207.90 & 236.62 \\
\hline & $D_{6}$ & & 278.49 & 273.63 & 312.64 \\
\hline \multirow[t]{6}{*}{$\mathrm{BW} 4=[23.4-24.7]$} & $D_{1}^{\circ}$ & & & 297.77 & 237.16 \\
\hline & $D_{2}$ & & & 390.22 & 503.03 \\
\hline & $D_{3}$ & & & 449.57 & 543.27 \\
\hline & $D_{4}$ & & & 658.97 & 777.45 \\
\hline & $D_{5}$ & 906.06 & & 932.45 & 1051.62 \\
\hline & $D_{6}$ & & 1409.00 & 1291.78 & 1285.76 \\
\hline
\end{tabular}

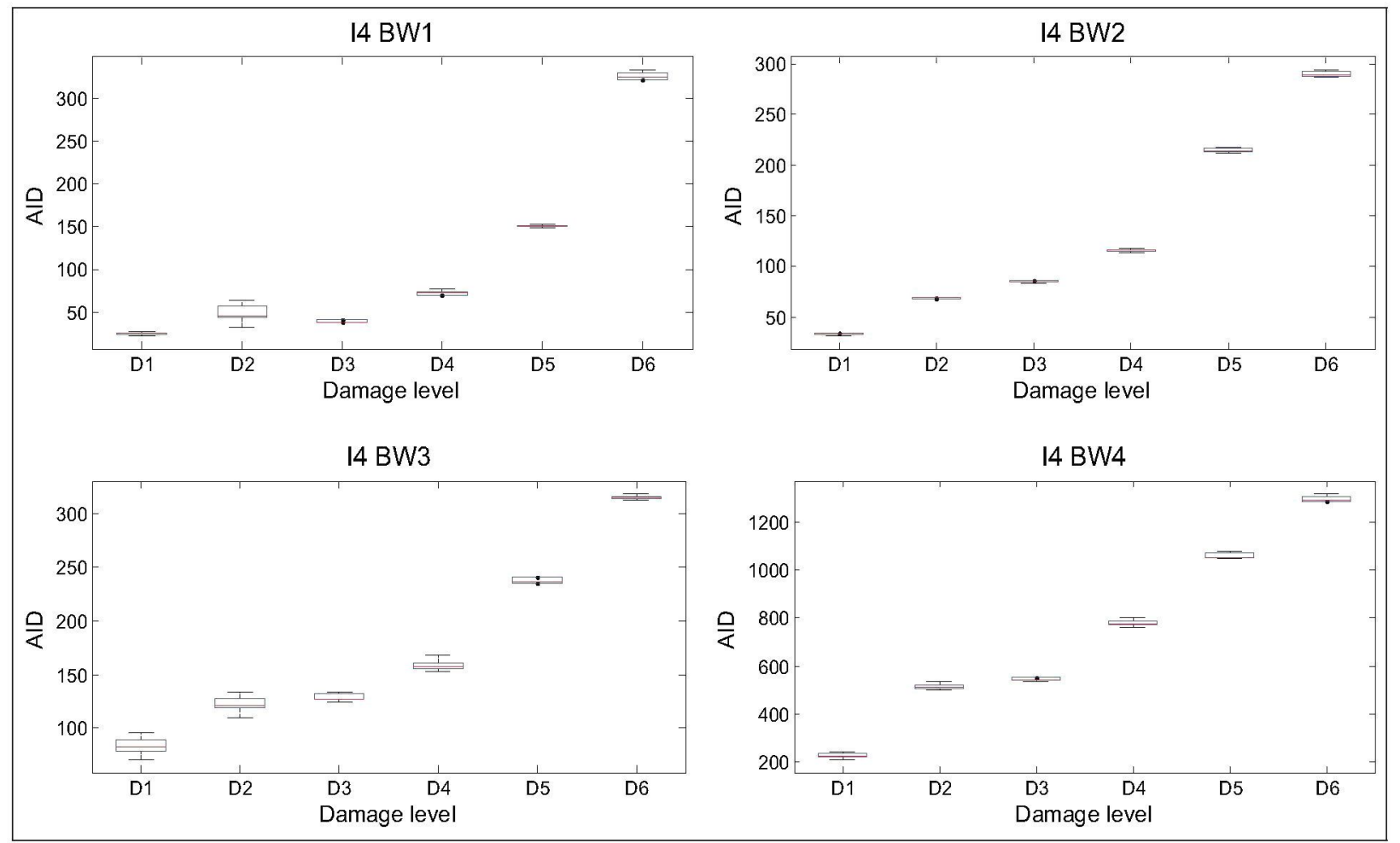

Figure I 4. Value of the AID for all the tests on specimen 14. The whiskers, in black color, show the maximum and minimum values obtained for AID in each test. The boxes have lines at the median, lower, and upper quartile values. AID: area index damage; BW: bandwidth. 


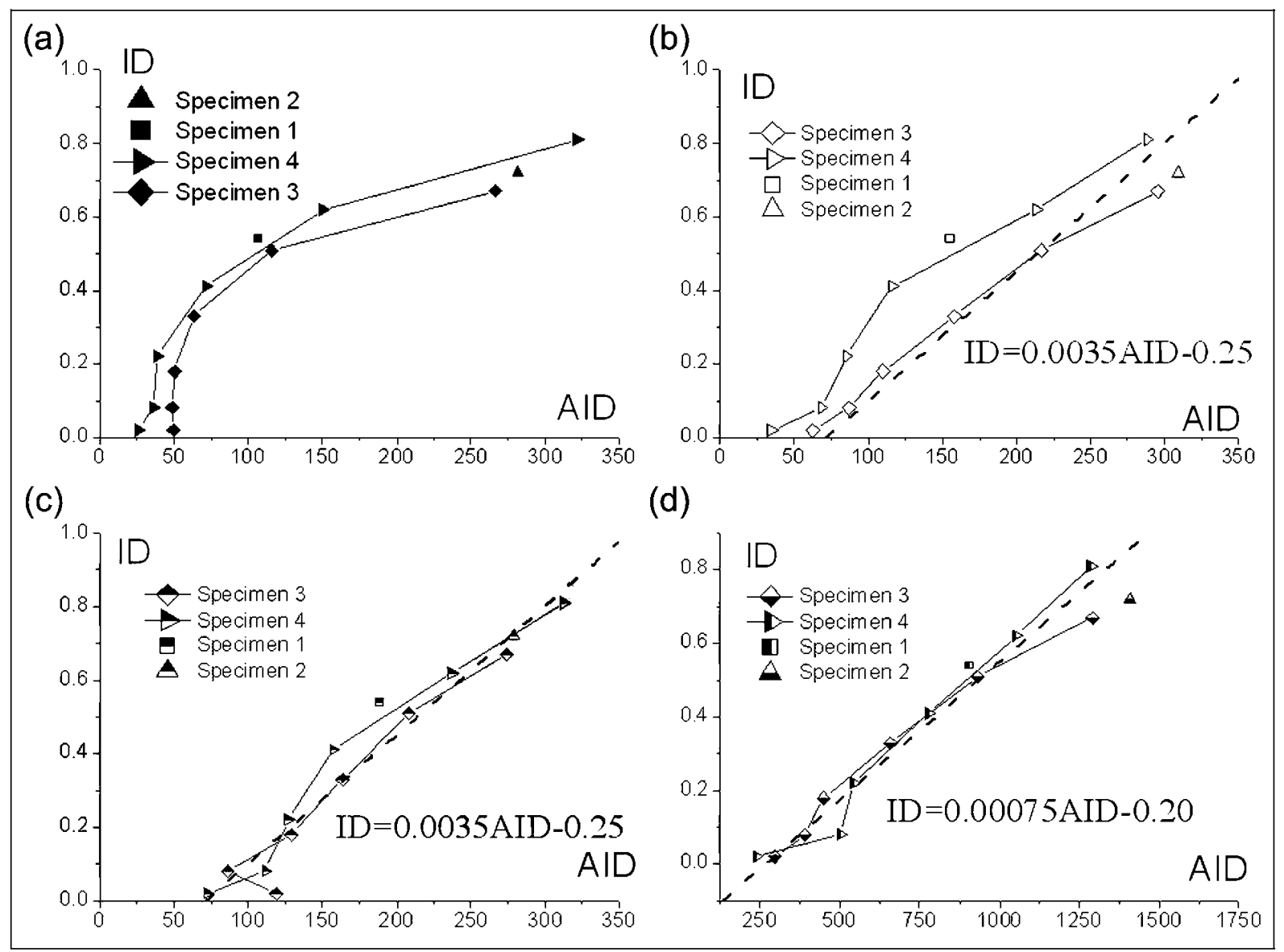

Figure I 5. Comparison between AID and ID in different frequency bands: (a) BWI: $1.4-I .7 \mathrm{kHz}$, (b) BW2: 4.4-4.7 kHz, (c) BW3: 9.4-9.7 kHz, and (d) BW4: 23.4-24.7 kHz.

AID: area index damage; ID: index of damage; BW: bandwidth.

monitor the damage on the EDDs under study. Once the index AID is calculated in this range, the corresponding index of mechanical damage ID can be readily obtained with equation (20).

Finally, in a real structure, the EDDs should be replaced when the damage quantified with the index ID and calculated from simple vibration tests with equations (19) and (20) approaches ID $=1$ (a reasonable limiting value would be ID $=0.75$ ). These vibration tests should be conducted after an earthquake or strong wind storm.

\section{Conclusion}

In this experimental study, a health monitoring technique to quantitatively evaluate the damage in hysteretic dampers subjected to cyclic loading is investigated. The type of damper under study consists of a segment of I-section steel profile. The technique uses nonparametric statistical processing of the excitation and response signals obtained from simple vibration tests conducted with two piezoelectric ceramic sensors glued to the web of the I-section.

The signals are processed with an FRF-based method, and the damage is detected from the changes in the frequency response of the specimen. From the feature quantity $Q$ obtained by mean estimation of FRF, a $|z|$-statistic is obtained. On the basis of the $|Z|$ statistic, this article proposes a new index, AID, which is found to be sensitive to the level of damage on the damper. The new index AID is compared with a mechanical index ID previously proposed in the literature and calibrated with the actual damage observed in the dampers. From cyclic and vibration tests conducted on four hysteretic dampers, the index AID is found to be closely correlated with the ID in several frequency bands through a straight line. As a result, simple formulae to predict ID from AID are proposed. This makes it possible to precisely monitor the damage on the damper and predict the proximity to failure from simple vibration tests, without resorting to cumbersome and expensive instrumentation on the damper. 


\section{Acknowledgements}

The authors appreciate the collaboration of Andrés RoldánAranda for his help in the experimental setup.

\section{Declaration of conflicting interests}

The authors declare that there is no conflict of interest.

\section{Funding}

This research received the financial support of the local government of Spain, Consejería de Innovación, Ciencia y Tecnologia, Projects P07-TEP-02610 and P08-TEP-03641. 